\title{
Subspace intersection using acoustic vector sensors by extracting main eigenspace of modal subspace
}

\author{
Weiwei $\mathrm{Ai}^{\mathrm{a}}$, Jinyu Xiong and Xiaoyong Zhang \\ Science and Technology on Blind Signal Processing Laboratory, 610041Chengdu, China
}

\begin{abstract}
The subspace intersection method using acoustic vector sensors [5] provides unbiased bearing estimates in a range-independent shallow ocean with the advantage of both subspace intersection(SI) method and acoustic vector sensors(AVS) array. But its robustness is poor. In this paper, an improved SI method based on extracting main eigenspace of modal subspace is proposed. Simulations show that the performance of the improved method in this paper is better than that of the improved method by compressing modal space with narrower main lobe width and lower estimation error.
\end{abstract}

Keywords: subspace intersection (SI); acoustic vector sensors (AVS); normal mode; main eigenspace.

\section{Introduction}

Bearing estimation is always a hard work in shallow water. Most of the traditional methods, which assume plane-wave propagation, lead to biased bearing estimates due to the multimode nature of acoustic wave propagation in the ocean. To solve this problem, Matched field processing (MFP) techniques was proposed by Gong [1], but this method works at the expense of computational complexity because of the three-dimensional search in the bearing-range-depth space. The subspace intersection method given by Lakshmipathi provides high-resolution bearing estimation based on a more appropriate normal mode propagation model [2]. SI alleviates the problems of both bias and computational complexity compared to MFP by a one-dimensional searching of bearing without range and depth. But the robustness of SI is poor because of the QR decomposition. Some methods were presented to solve this problem such as least square subspace intersection [3] and compressing the modal space subspace intersection [4]. Nagananda [5] extend SI to an AVS array which measure acoustic pressure as well as all components of particle velocity, and AVS array get better performance than scalar array, but stability is not improved.

After studying SI method, we find that the modal subspace of different bearing have strong correlation when the number of the normal modes rises. So extracting main eigenspace SI (EMS_SI) method based on acoustic vector sensors, which can not only make sure the eigenspace matrix is singular but also reduce the correlation of modal subspace, is proposed in this paper. Simulations show that the performance of EMS_SImethod is better than that of the improved method by compressing modal subspace (CMS_SI) [4] with narrower main lobe width and lower estimation error.

\footnotetext{
${ }^{a}$ Corresponding author : aww_101@163.com
} 


\section{AVS array data model based on normal mode propagation model}

The AVS array data model in shallow ocean usedin this paper isthe same with that described in [5]. A horizontal linear AVS array of $N$ sensors at depth $z_{a} \mathrm{~m}$ is taken into account, and the inter-sensor spacing isd. There are $J$ narrowband sources of centre frequencyf, which are located at depth $z_{j} \mathrm{~m}$, ranges $r_{j} \mathrm{~m}$ and bearing $\theta_{j} j=1, \ldots, J$. Bearing is measured with respect to the end-fire direction of the array.

The output of AVS array could be expressed as

$$
\mathbf{X}(t)=\mathbf{P}(\boldsymbol{\Phi}) \mathbf{S}(t)+\mathbf{n}(t)
$$

where $\mathbf{n}$ is the array noise vector and

$$
\begin{gathered}
\boldsymbol{\Phi}=[\boldsymbol{\theta}, \mathbf{r}, \mathbf{z}]^{T} \\
\boldsymbol{\theta}=\left[\theta_{1}, \ldots, \theta_{J}\right]^{T} \\
\mathbf{r}=\left[r_{1}, \ldots, r_{J}\right]^{T} \\
\mathbf{z}=\left[z_{1}, \ldots, z_{J}\right]^{T}
\end{gathered}
$$

are the vector of unknown parameters, and

$$
\mathbf{S}(t)=\left[s_{1}(t), s_{2}(t), \cdots, s_{J}(t)\right]^{T}
$$

is the source signal vector, and $\mathbf{P}(\boldsymbol{\Phi})$ is a matrix defined as

$$
\mathbf{P}(\boldsymbol{\Phi})=\mathbf{A}(\boldsymbol{\theta}) \mathbf{B}(\mathbf{r}, \mathbf{z})
$$

and

$$
\begin{gathered}
A(\boldsymbol{\theta})=\left[\boldsymbol{A}\left(\theta_{1}\right), \ldots, \boldsymbol{A}\left(\theta_{J}\right)\right] \\
\boldsymbol{B}(\boldsymbol{r}, \mathbf{z})=\operatorname{diag}\left(\boldsymbol{b}\left(r_{1}, z_{1}\right), \ldots, \boldsymbol{b}\left(r_{J}, z_{J}\right)\right) \\
\mathbf{A}\left(\theta_{j}\right)=\left[\mathbf{a}_{1}\left(\theta_{j}\right), \mathbf{a}_{2}\left(\theta_{j}\right), \cdots, \mathbf{a}_{M}\left(\theta_{j}\right)\right] \\
\mathbf{b}\left(r_{j}, z_{j}\right)=\left[b_{1}\left(r_{j}, z_{j}\right), \ldots, b_{M}\left(r_{j}, z_{j}\right)\right] \\
b_{m}\left(r_{j}, z_{j}\right)=B_{0} \psi_{m}\left(z_{a}\right) \psi_{m}\left(z_{j}\right)\left[\frac{e^{i k_{m} r_{j}-\delta_{m} r_{j}}}{\sqrt{k_{m} r_{j}}}\right]
\end{gathered}
$$

In these formulas, $M$ is the total number of the normal modes, and $B_{0}$ is a complex quantity independent of $r_{j}, z_{j}, \theta_{j}, z_{a}$. For AVS array, 


$$
a_{m}\left(\theta_{j}\right)=\left[1, e^{j k_{m} d \cos \theta_{j}}, \cdots, e^{j(N-1) k_{m} d \cos \theta_{j}}\right]^{T} \otimes\left[1, \frac{k_{m} \cos \left(\theta_{j}\right)}{k}, \frac{k_{m} \sin \left(\theta_{j}\right)}{k}, \frac{j \psi_{m}^{\prime}\left(z_{\mathrm{j}}\right)}{k^{*} \psi_{m}\left(z_{j}\right)}\right]^{T}
$$

$\psi_{m}(z)$ and $k_{m}$ are the eigenfunction and wavenumber of the $m$ th normal mode.

Considering the sound sources $\mathbf{S}(t)$ are uncorrelated white Gaussian noise with variances $\sigma_{j}^{2}$, and the sound sources $\mathbf{S}(t)$ are uncorrelated with the noise $\mathbf{n}(t)$, and $\mathbf{n}(t)$ is uncorrelated white Gaussian noise with variances $\sigma_{n}^{2}$.

Since correlation matrix of noiseis not diagonal, so it needs to be whitened before signal processing. In order to satisfy this condition, we apply the whitening transformation $\mathbf{n}_{\mathbf{w}}=\mathbf{W} * \mathbf{n}$, where the matrix $\mathbf{W}$ is given in [5]. So we get

$$
\mathbf{X}_{w}(t)=\mathbf{W}^{*} \mathbf{X}(t)
$$

and the correlation matrix of $\mathbf{X}_{w}(t)$ is defined as

$$
\mathbf{R}_{w}=E\left[\mathbf{X}_{w}(t) \mathbf{X}_{w}(t)^{H}\right]
$$

In practical applications, we use the following formula to estimate the true correlation matrix

$$
\hat{\mathbf{R}}_{w}=\frac{1}{L} \sum_{t=1}^{L}\left[\mathbf{X}_{w}(t) \mathbf{X}_{w}(t)^{H}\right]
$$

where $L$ is the number of snapshots.

\section{Review of SI method based on AVS array}

The idea of SI method based on AVS is concise [5]. Suppose $\mathbf{U}_{s}=\operatorname{span}\left\{\mathbf{W} \mathbf{p}\left(\varphi_{1}\right), \cdots, \mathbf{W} \mathbf{p}\left(\varphi_{J}\right)\right\}$ is the signal subspace and $\mathbf{A}_{s}(\theta)=\operatorname{span}\left\{\mathbf{W a}\left(\theta_{1}\right), \cdots, \mathbf{W a}\left(\theta_{M}\right)\right\} \quad$ is the modal subspace. Then $\mathbf{U}_{s} \cap \mathbf{A}_{s}(\theta) \neq \varnothing$ if and only if $\theta \in\left\{\theta_{1}, \cdots, \theta_{J}\right\}$. Signal subspace could be obtained by decomposing the covariance matrix:

$$
\boldsymbol{R}_{w}=\boldsymbol{W P} \boldsymbol{R}_{s} \boldsymbol{P}^{H} \boldsymbol{W}^{H}+\sigma_{N}^{2} \boldsymbol{I}
$$

Assume $\mathbf{R}_{s}$ is a full rank matrix. Let $u_{1}, \cdots, u_{4 N}$ indicate the unit-norm eigenvectors of $\mathbf{R}_{w}$ arranged in a descending order with respect to the value of their eigenvalues. Thus, $\mathbf{U}_{s}=\operatorname{span}\left\{u_{1}, \cdots, u_{J}\right\}$. The SI method based on AVS could be described as follows:

(1) Whiten the array noise by using $\boldsymbol{W}$;

(2) Estimate the covariance matrix $\mathbf{R}_{w}$ from samples;

(3) Construct the signal space $\mathbf{U}_{s}$ through eigen decomposition of $\mathbf{R}_{w}$;

(4) Construct $\mathbf{D}=\left[\mathbf{A}_{s}(\theta), \mathbf{U}_{s}\right]$ and do the $\mathrm{QR}$ decomposition to get $\mathbf{D}=\mathbf{Q R}$. Let $r_{j j}$ indicate the diagonal elements of $\mathbf{R}$;

(5) Compute the function $B_{S I}(\theta)=\left[\min _{M+1 \leq j \leq M+J} r_{j j}^{2}\right]^{-1}$.

The anglescorresponding to thepeaks of $B_{S I}(\theta)$ are the bearing of sources.

\section{Extracting main eigenspace SI method based on AVS}


The original SI method needs to do QR decomposition of matrix D. It is not stable because the matrix D especially $\mathbf{A}_{s}(\theta)$ is singular. By studying the SI method based on AVS, there is another reason that impacts on the performance of SI. For a given channel, the number of propagating modes $M$ depends on the signal frequency. In general, $M$ increases as the frequency is increased. The modal matrix $\mathbf{A}(\theta)$ is $4 N^{*} M$. When $M$ increases, the number of column vector of $\mathbf{A}(\theta)$ increases. Then, the modal subspace of different directions have strong correlation, leading to main lobe width wider.

To solve the problem above, the method of extracting main eigenspace of modal subspace is proposed in this paper. Ideas as follows:

Construct Hermitian matrix:

$$
\mathbf{R}_{A}(\theta)=\mathbf{A}(\theta) \mathbf{A}^{H}(\theta)
$$

Eigendecompose $\mathbf{R}_{A}(\theta)$

$$
\mathbf{R}_{A}(\theta)=\mathbf{U}_{A}(\theta) \Lambda \mathbf{U}_{A}^{H}(\theta)
$$

where

$$
\boldsymbol{\Lambda}=\operatorname{diag}\left\{\mu_{1}, \cdots, \mu_{4 N}\right\}
$$

is a diagonal matrix, and diagonal elements are the eigenvalue of $\mathbf{R}_{A}(\theta)$, and they are arranged in a descending order.

According to the theory of signal space, the modal subspace $\mathbf{A}_{s}(\theta)$ is the same with the space $\mathbf{U}_{C M S}=\operatorname{span}\left\{\mathbf{u}_{a 1}, \cdots, \mathbf{u}_{a r}\right\}$, where $r=\operatorname{rank}(\mathbf{A}(\theta))$ and $\mathbf{u}_{a n}(n=1, \ldots 4 N)$ are the column vectors of $\mathbf{U}_{A}(\theta)$, and $\mathbf{U}_{C M S}$ is singular. So we can use $\mathbf{U}_{C M S}$ instead of $\mathbf{A}_{s}(\theta)$ [4]. After analyzing the eigenvalue $\mu_{n}(n=1, \ldots r)$ of $\mathbf{R}_{A}(\theta)$, some of them are small, and the eigenvectors corresponding to them have little effect on $\mathbf{U}_{\text {CMS }}$. So we bring in the threshold $\gamma$ to extracting main eigenspace of modal subspace:

$$
\mathbf{U}_{E M S}(\theta)=\left\{\mathbf{u}_{a 1}(\theta), \cdots, \mathbf{u}_{a N_{A}}(\theta)\right\}\left(\frac{\mu_{N_{A}}}{\mu_{1}}>\gamma, \frac{\mu_{N_{A}+1}}{\mu_{1}}<\gamma\right)
$$

where $N_{A}$ is the number of main eigenvectors chosenafter setting the threshold $\gamma$.

In this way, $\mathbf{U}_{E M S}(\theta)$ that we construct is singular, and importantly, the extracted modal subspace of different directions have lower correlation. 


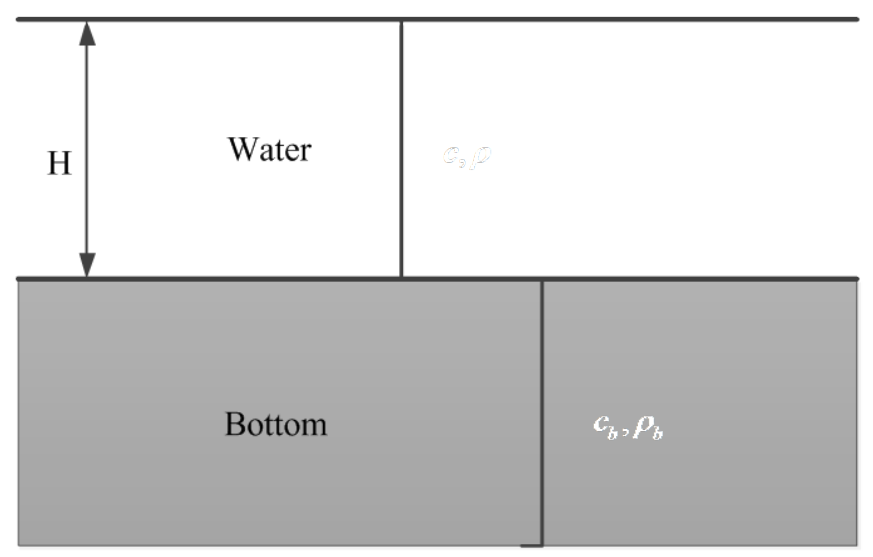

Figure1. Ocean model

\section{Simulation}

We consider the Pekeris Ocean model as [5]. The ocean model is shown in Figure1.The channel parameters are assumed to be the following: water depth $H=75 \mathrm{~m}$, sound speed in water $c=1500 \mathrm{~m} / \mathrm{s}$, sound speed in bottom $c_{b}=1700 \mathrm{~m} / \mathrm{s}$, density of water $\rho=1 \mathrm{~g} / \mathrm{cm}^{3}$, density of bottom $\rho_{b}=1.5 \mathrm{~g} / \mathrm{cm}^{3}$, attenuation in bottom $\delta=0.5 \mathrm{~dB} / \lambda$ where $\lambda=c / f$, and $f=500 \mathrm{~Hz}$. All the sources are located at ranges $r=5000 \mathrm{~m}$ and depth $z_{j}=37.5 \mathrm{~m}$. The acoustic vector sensors array are located at depth $z=5 \mathrm{~m}$, and the inter-sensor spacing $d=\lambda / 2$, the number of sensors $N=20$. All the results have been obtained by averaging over 200 simulations, and the snapshots is $L=350$. The signal-to-noise ratio for the source is defined as [5].

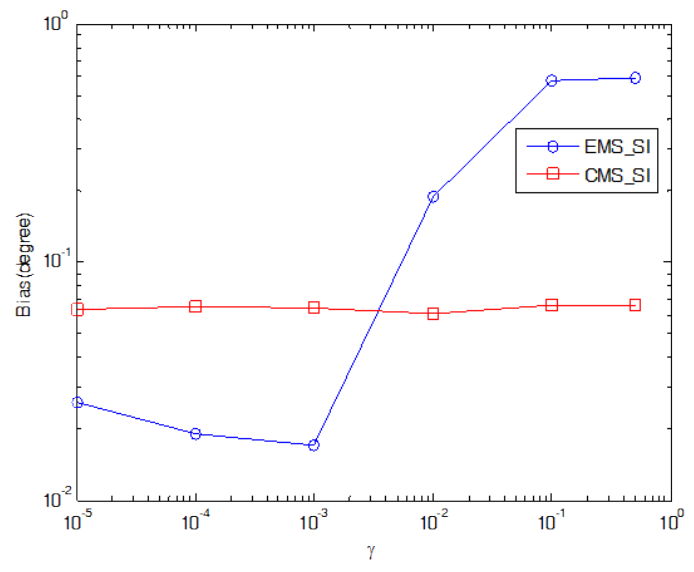

Figure 2. Bias vs $\gamma$ for EMS_SI and CMS_SI. One source at $\theta=60$, SNR $=15 \mathrm{~dB}$ 


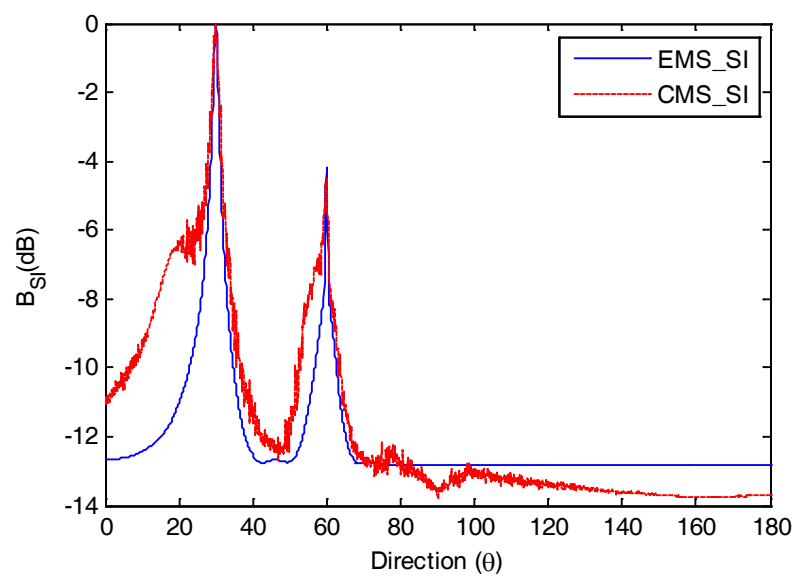

Figure 3. Response function for EMS_SI and CMS_SI. Two sources at $\theta=30,60$, SNR $=15 \mathrm{~dB}$

In order to choose the proper parameter $\gamma$, we simulate $\gamma$ vs bias of EMS_SI for $\theta=60$, $\mathrm{SNR}=15 \mathrm{~dB}$, and compare the result with that of CMS_SI. Simulation result is show in Figure2. It is shown that, for EMS_SI, the bias of bearing estimates is the least at $\gamma=0.001$, and the performance of EMS_SI is better than that of CMS_SI when $\gamma$ is not too big, $\gamma=0.001$ included absolutely. The simulation results in the following paper are obtained under the condition of $\gamma=0.001$.

EMS_SI response function $B_{S I}(\theta)$ is compared with CMS_SI in Figure3 for $\theta=30,60$, SNR $=15 \mathrm{~dB}$. It is seen that, both EMS_SI and CMS_SI can realize the accurate bearing estimation in normal mode propagation model, and EMS_SI has narrower main lobe width than CMS_SI.

Figure 4 and 5 show the plots of bias vs SNR and the plots of root mean square error (RMSE) vs SNR for both EMS_SI and CMS_SI, and $\theta=60^{\circ}$. It is seen that, when SNR changes from -5dB to 20dB, EMS_SI performs better than CMS_SI obviously, especially under low SNR environment.

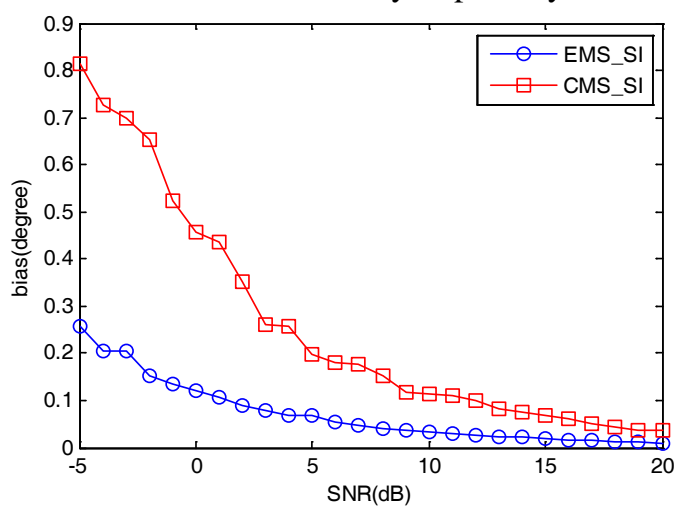

Figure 4. Bias vs SNR for EMS_SI and CMS_SI.One source at $\theta=60^{\circ}$ 


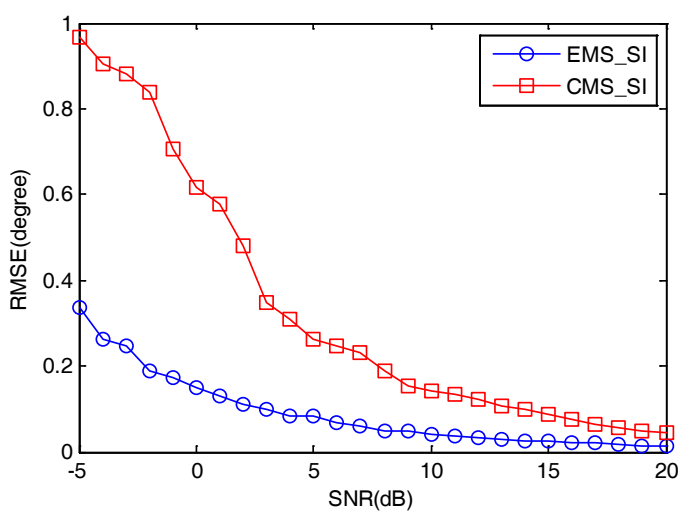

Figure 5. RMSE vs SNR for EMS_SI and CMS_SI. One source at $\theta=60^{\circ}$

Figure 6 and 7 show the plots of bias vs bearing angle $\theta$ and the plots of RMSE vs bearing angle $\theta$ for both EMS_SI and CMS_SI, and SNR $=15 \mathrm{~dB}$. It is seen that, for both EMS_SI and CMS_SI, the bias and RMSE decrease as $\theta$ approaches $90^{\circ}$, but EMS_SI performs better than CMS_SI.

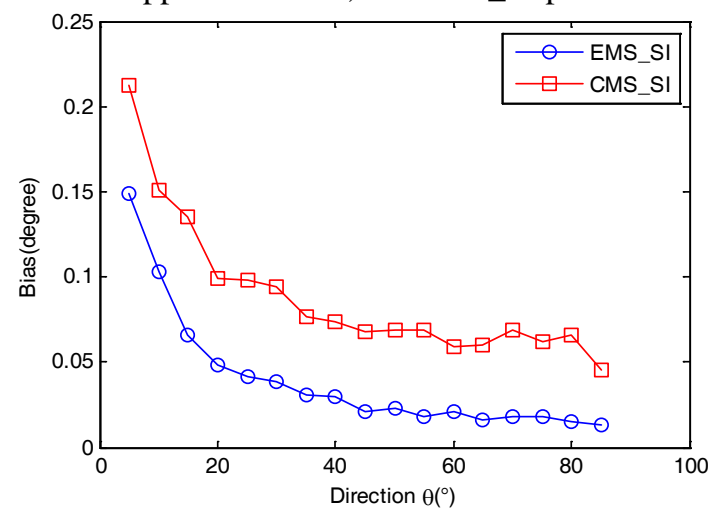

Figure 6. Bias vs SNR for EMS_SI and CMS_SI. One source is set and SNR=15dB

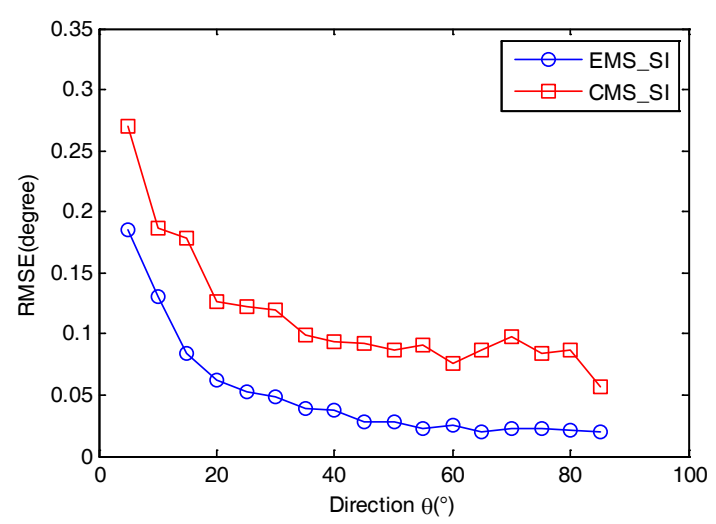

Figure 7. RMSE vs SNR for EMS_SI and CMS_SI. One source is set and SNR=15dB

\section{Conclusion}

In this paper, we have presented the extracting main eigenspace SI method based on the compressing eigenspace SI method using a horizontal linear array of acoustic vector sensors. In this method, we use the main eigenspace extracted from modal subspace instead of the modal subspace,and the correlation 
of modal subspacein different direction is reduced, in this way, we can make the SI method more stable.Simulation results show that the main lobe width for EMS_SI is narrower than CMS_SI, and the bias and RMSE for EMS_SI are significantly lower than those for CMS_SI, especially at low SNR. No matter how the target's direction changes, the bias and RMSE for EMS_SI are significantly lower than those for CMS_SI.

\section{References}

1. ZaixiaoGong, Jing Lin, LianghaoGuo, The effect of acoustic waves' phase speed on preciseness of DOA estimation in shallow water, Acta Acustica,27, 492-496 (2002)

2. S.Lakshmipathi, G. V. Anand, Subspace intersection method of high-resolution bearing estimation in shallow ocean, Signal Processing, 84, 1367-1384(2004)

3. Jincheng Pang, Jing Lin, Aimin Zhang, and Xiaodi Huang, Subspace intersection method of bearing estimation based on least square approach in shallow ocean in International Conference on Acoustics, Speech, \& Signal Processing.IEEE,27, 2433-2436(2008)

4. Liang Wang, Zhijie Song, A stable subspace intersection method on long line array,Technical Acoustics, 27, 426-427 (2008)

5. K. G. Nagananda, G. V. Anand, Subspace intersection method of high-resolution bearing estimation in shallow ocean using acoustic vector sensors,Signal Processing,84, 1367-1384 (2010) 